\title{
On the Estimation of Randomly Sampled 2D Spatial Fields under Bandwidth Constraints
}

\author{
Javier Matamoros, Member, IEEE, Flavio Fabbri, Student Member, IEEE, \\ Carles Antón-Haro, Senior Member, IEEE, and Davide Dardari, Senior Member, IEEE
}

\begin{abstract}
In this paper, we address the problem of the estimation of a spatial field defined over a two-dimensional space with wireless sensor networks. We assume that the field is (spatially) bandlimited and that it is sampled by a set of sensors which are randomly deployed in a given geographical area. Further, we impose a total bandwidth constraint which forces the quantization error in the sensor-to-FC (Fusion Center) channels to depend on the actual number of sensors in the network. With these assumptions, we derive an analytical expression of the mean-square error (MSE) in the reconstructed random field and, on that basis, an approximate closed-form expression of the optimal sensor density which attains the best trade-off in terms of observation, sampling and quantization noises. The analysis is carried out both in Gaussian and Rayleigh-fading scenarios without transmit Channel State Information (CSI). For the latter scenario, we also derive an expression of the common and constant rate at which the observations must be quantized. Computer simulation results illustrate the dependency of the optimal operating point on the variance of the observation noise or the signal-to-noise ratio in the sensor-to-FC channels, as well as the scaling law of the reconstruction MSE (which is also derived analytically) for both scenarios.
\end{abstract}

Index Terms-Distributed estimation, wireless sensor networks, random sampling.

\section{INTRODUCTION}

$\mathbf{I}$ $\mathrm{N}$ recent years, research on Wireless Sensor Networks (WSNs) has attracted considerable attention. This is in part motivated by the large number of applications envisaged, which encompasses environmental monitoring, building automation, supervision and control of energy grids, or inventory tracking, to name a few [1], [2].

In this paper, we focus our attention on environmental monitoring applications. Specifically, our goal is to estimate a spatial field (e.g., moisture, temperature) in a given geographical area from the samples collected by a large number of low-cost, battery-powered sensor nodes. The observations are

Manuscript received November 11, 2010; revised February 16 and June 6, 2011; accepted August 1, 2011. The associate editor coordinating the review of this paper and approving it for publication was S. Cui.

J. Matamoros and C. Antón-Haro are with the Centre Tecnologic de Telecomunicacions de Catalunya, Av. Carl Friedrich Gauss, 7 (Building B4) 08860, Castelldefels, Spain (e-mail: \{javier.matamoros, carles.anton\}@cttc.es).

F. Fabbri is with WiLAB - DEIS, University of Bologna, V.le Risorgimento 2, I-40136, Bologna (BO), Italy (e-mail: flavio.fabbri@unibo.it).

D. Dardari is with WiLAB - DEIS, University of Bologna at Cesena Campus, via Venezia 52, I-47521, Cesena (FC), Italy (e-mail: ddardari@ieee.org).

This work is partly supported by the European Commission through the project NEWCOM++ (216715), the Generalitat de Catalunya (2009 SGR 1046), and the Spanish Ministry of Science and Innovation through the project JUNTOS (TEC2010-17816).

Digital Object Identifier 10.1109/TWC.2011.092911.102015 conveyed via wireless links to a Fusion Center (FC) which, ultimately, is charge of estimating (i.e. reconstructing) the entire spatial field. The reconstruction error is mainly affected by (i) the quality of the received samples; (ii) the number of spatial samples; and (iii) the spatial sampling pattern. The quality of the received samples depends both on the observation noise in the sensing device (a technological issue and, as such, out of the scope of this paper) and the encoding scheme at the sensor node. The encoding/decoding process can definitely benefit from any prior statistical knowledge on the spatial field, such as its spatial correlation model (e.g. rational quadratic, Matérn or, power exponential [3][6]), source model (e.g. Gaussian Markov), or dynamic range. For example, this can be used in order to remove correlation in adjacent spatial samples and, by doing so, increase the energy efficiency of transmissions [7]. On the contrary, other works barely exploit prior statistical knowledge in an attempt to keep the framework as general as possible. For instance, the probabilistic quantization scheme and power scheduling strategy of [8] only makes mild assumptions on the dynamic range of the parameter of interest. Similarly, [9], [10] investigate universal (pdf-unaware) estimators of spatiallyhomogeneous parameters under strict bandwidth constraints (1 bit per sensor).

The fact that wireless networks typically operate under a total bandwidth constraint, render the aforementioned issues (i) and (ii) intertwined. The so-called "bit-conservation principle" [11] states that, for spatially bandlimited processes allowing inter-sensor communication, the bit budget per Nyquistperiod can be arbitrarily used to (i) increase the quantization precision; or (ii) add more sensor nodes, while retaining the same decay profile of the reconstruction error. In this context, we showed in [12] that for (one-dimensional) bandwidthand power-constrained WSNs there exists an optimal number of sensor nodes which minimizes the actual error in the reconstructed random field (i.e. it attains the optimal balance between interpolation and quantization errors). The authors in [13] address the problem of distributed estimation of Gaussian Markov fields under stringent bandwidth constraints, where each sensor quantizes its observation with one bit.

As for the sampling pattern, most of the works on spatial field estimation adopt deterministic ones (i.e. equally-spaced sensors). However, this is difficult to achieve in practice, in particular for deployments in large or remote areas. In these scenarios, it is more realistic to conduct an airdrop sensor deployment which results into a random sampling pattern. In 
this line, [14] analyzes the impact of such random sampling patterns on the reconstructed random field in a rather idealized scenario where quantization errors are not taken into account. Besides, in [4] Tong et al conclude that, whereas deterministic sampling pays off in the high-SNR regime, both schemes exhibit comparable performance in the low-SNR regime.

Contribution: In this paper, we address the problem of the estimation of bandlimited spatial field. Unlike in [4]-[6], [13], [15], we do not exploit any prior statistical knowledge on the spatial field, which includes any correlation information or model. We go one step beyond the 1-D case in our previous work [12] and embrace the (more general) case of the estimation of a two-dimensional spatial field. Further, we adopt a random sampling strategy (a deterministic sampling pattern was used in [12]). Unlike in [14], we impose a total bandwidth (and sum-power) constraint for the sensor-to-FC channels and, consequently, the impact of quantization errors need to be taken into account in the optimization process. With these assumptions, we ask ourselves whether increasing the sensor density is always worth doing (in terms of reconstruction error) or if, alternatively, an optimal operational point exists. To answer this question, we derive an approximate closed-form expression of the optimal sensor density for two scenarios of interest, namely, Gaussian and Rayleigh-fading channels in the sensor-to-FC links. For this latter scenario, we assume that sensors operate without transmit CSI and, hence, the encoding rate cannot be dynamically adjusted. We derive an analytical expression of the common (to all sensors) and constant (over time) rate which is optimal in terms of reconstruction error. Finally, we derive the scaling law of the reconstruction MSE in the high-SNR regime for both scenarios.

This paper is organized as follows. First, in Section II, we introduce the signal, communication and system model. Next, Section III provides a general framework for the analysis of the distortion in the reconstructed random field. In Section IV, we particularize the aforementioned distortion analysis for Gaussian and Rayleigh-fading scenarios and, on that basis, we derive closed-form expressions of the optimal sensor density and/or encoding rate. In Section V, we present some computer simulations and numerical results and, finally, we close the paper by summarizing the main findings in Section VI.

Notation: Throughout this paper, $\mathbf{s}$ stands for the $l$ dimensional spatial variable. Random processes are denoted by uppercase letters (e.g., $Y(\mathbf{s}), X_{s}(\mathbf{s})$ ) whereas lowercase is used for deterministic signals or individual realizations (e.g., $z(\mathbf{s}))$. The norm operator is denoted by $\|\cdot\|$.

\section{Signal, Communication and System Model}

\section{A. Spatial Field Model}

Let $z(\mathbf{s})$ denote a field defined over an $l$-dimensional space ${ }^{1}$. We assume that $z(\mathbf{s})$ is spatially bandlimited, i.e., it does not have significant spectral components beyond $B_{z}$ which denotes the bandwidth per dimension of $z(\mathbf{s})$. The part of spatial field monitored by the WSN is contained in a ball $\mathcal{A}$

\footnotetext{
${ }^{1}$ Here $z(\mathbf{s})$ is a deterministic function of $\mathbf{s}$. We could eventually think of $z(\mathbf{s})$ as a realization of a random process $Z(\mathbf{s})$ however, no assumption on the statistical nature of $Z(\mathbf{s})$ (and hence of $X(\mathbf{s})$ ) is made.
}

of radius $R$ and Lebesgue measure $\mu(\mathcal{A})=A$. Hence, the truncated signal of interest is given by

$$
x(\mathbf{s})=z(\mathbf{s}) \cdot r_{A}(\mathbf{s})
$$

with

$$
r_{A}(\mathbf{s})=\left\{\begin{array}{rr}
1 & \mathbf{s} \in \mathcal{A} \\
0 & \text { otherwise }
\end{array}\right.
$$

and it is assumed to be of finite energy $E_{x}$. We denote the $l$-dimensional Fourier transform, $S_{x}(\boldsymbol{\nu})$, the autocorrelation function, $R_{x}(\boldsymbol{\tau})$, and the energy spectral density, $\mathcal{E}_{x}(\boldsymbol{\nu})$, of $x(\mathbf{s})$, by

$$
\begin{aligned}
S_{x}(\boldsymbol{\nu}) & =\mathfrak{F}^{(l)}[x(\mathbf{s})] ; \\
R_{x}(\boldsymbol{\tau}) & =\int_{\Re^{l}} x(\mathbf{s}) x(\mathbf{s}-\boldsymbol{\tau}) d \mathbf{s} ; \\
\mathcal{E}_{x}(\boldsymbol{\nu}) & =\mathfrak{F}^{(l)}\left[R_{x}(\boldsymbol{\tau})\right],
\end{aligned}
$$

respectively, where $\boldsymbol{\tau}=\left(\tau_{1}, \tau_{2}, \ldots, \tau_{l}\right)$, and $\boldsymbol{\nu}=\left(\nu_{1}, \nu_{2}, \ldots, \nu_{l}\right)$ is the vector of spatial frequencies. The Fourier transform of $x(\mathbf{s})$ can also be expressed as

$$
S_{x}(\boldsymbol{\nu})=S_{z}(\boldsymbol{\nu}) \otimes R_{A}(\boldsymbol{\nu}),
$$

where $R_{A}(\boldsymbol{\nu})=\mathfrak{F}^{(l)}\left[r_{A}(\mathbf{s})\right]$ and $\otimes$ stands for the convolution operator. Note that, due to the spatial truncation of the original signal, $x(\mathbf{s})$ is not bandlimited. However, it can be easily verified that, in general, $R_{A}(\boldsymbol{\nu}) \approx 0$ when $\|\boldsymbol{\nu}\|>\frac{1}{\pi R}$. In the practical two-dimensional case $(l=2)$, for instance, we have

$$
R_{A}(\boldsymbol{\nu})=\frac{R}{\|\boldsymbol{\nu}\|} J_{1}(2 \pi R\|\boldsymbol{\nu}\|),
$$

where $J_{1}($.$) is the Bessel Function of the first kind and order$ one. Consequently, $S_{x}(\boldsymbol{\nu})$ and, likewise, $\mathcal{E}_{x}(\boldsymbol{\nu}) \approx 0$ outside $\mathcal{S}=\left\{\boldsymbol{\nu}\right.$ s.t. $\left(-B<\nu_{1}<B,-B<\nu_{2}<B, \ldots,-B<\right.$ $\left.\left.\nu_{l}<B\right)\right\}$, with $B=\frac{1}{\pi R}+B_{z}$ in the two-dimensional case. The Lebesgue measure of $\mathcal{S}$ is $\mu(\mathcal{S})=\beta$, where $\beta=(2 B)^{l}$ represents the minimum Nyquist sampling rate in the case of deterministic (uniform) sampling of $x(\mathbf{s})$ [16], [17]. In practical applications, we have $\frac{1}{\pi R} \leq B_{z}$, that is, the area of observation is chosen larger than the typical spatial correlation distance which is proportional to $1 / B_{z}$.

\section{B. Random Sampling of Spatial Fields}

Departing from deterministic sampling patterns, we consider a scenario where nodes are randomly deployed according to a two-dimensional homogeneous Poisson Point Process (PPP) with spatial density $\rho$ (see Fig. 1). The stationary random sampling process $P(\mathbf{s})$ can thus be expressed as

$$
P(\mathbf{s})=\sum_{k} \delta\left(\mathbf{s}-\mathbf{s}_{k}\right)
$$

where $\delta($.$) is the Dirac pseudo-function, \mathbf{s}_{k}$ denotes the spatial location of the $k$-th sensor node ${ }^{2}$, and $P(\mathbf{s})$ verifies $\mu_{P}=$ $\mathbb{E}\{P(\mathbf{s})\}=\rho$. The statistical autocorrelation function and power spectral density reads [16], [18]

$$
R_{P}(\boldsymbol{\tau})=\rho \cdot \delta(\boldsymbol{\tau})+\rho^{2}
$$

\footnotetext{
${ }^{2}$ By construction, the spatial distribution of sensor nodes in a Point Poisson (sampling) Process is uniform in any ball $\mathcal{A}$.
} 


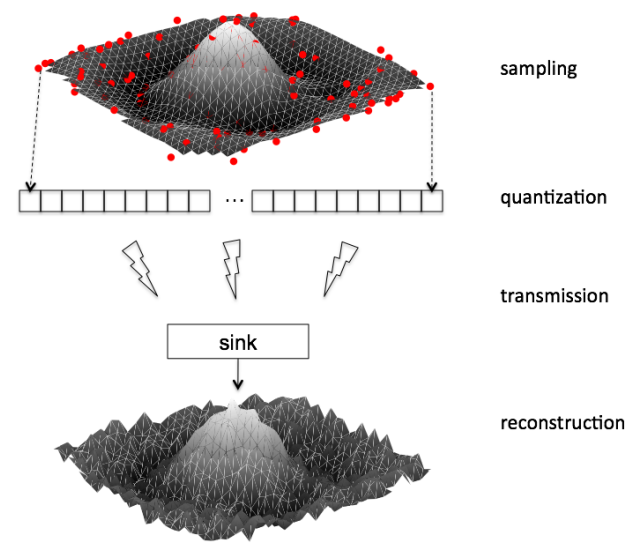

Fig. 1. Signal, communication and system model.

$$
S_{P}(\boldsymbol{\nu})=\rho+\rho^{2} \cdot \delta(\boldsymbol{\nu}),
$$

respectively. The total number $K$ of sensor nodes in a given observation area $A$, which follows from the associated Poisson Counting Process (i.e., the integral of $P(\mathbf{s})$ ), turns out to be a random variable (r.v.) with expectation $\mathbb{E}\{K\}=\rho A$ and Poisson distribution given by [1]

$$
p_{K}(k)=\operatorname{Pr}\{K=k\}=\frac{(\rho A)^{k}}{k !} e^{-\rho A} .
$$

We assume that sensor observations are corrupted by additive noise that, in the sequel, will be modeled as zero-mean independent and identically-distributed (i.i.d.) Gaussian random variables. Thus, the observed (noisy) version of the sampled spatial field reads

$$
R(\mathbf{s})=x(\mathbf{s}) \cdot P(\mathbf{s})+N(\mathbf{s}) \triangleq X_{s}(\mathbf{s})+N(\mathbf{s})
$$

where $X_{s}(\mathbf{s}) \triangleq \sum_{k} x_{k} \delta\left(\mathbf{s}-\mathbf{s}_{k}\right)$ with $x_{k}=x\left(\mathbf{s}_{k}\right)$; and $N(\mathbf{s}) \triangleq \sum_{k} n_{k} \delta\left(\mathbf{s}-\mathbf{s}_{k}\right)$ with $n_{k} \sim \mathcal{N}\left(0, \eta_{k}^{2}\right)$. Likewise, we can also write $R(\mathbf{s}) \triangleq \sum_{k} r_{k} \delta\left(\mathbf{s}-\mathbf{s}_{k}\right)$. From this model, it follows that the average observation signal-to-noise ratio (OSNR) at the $k$ th sensor node is given by $\Gamma_{\text {obs }, k} \triangleq E_{x} / \eta_{k}^{2}$.

\section{Quantization and Transmission}

Sensors adopt a uniform quantization scheme with subtractive dithering [19]. Specifically, each sensor uniformly quantizes the input signal $r_{k}$ using $L_{k}=2^{m_{k}}$ quantization levels and generates a discrete message $y_{k}=y_{k}\left(r_{k}, m_{k}\right)$ of $m_{k}$ bits. The actual value encoded in the discrete message can be modeled as

$$
y_{k}=x_{k}+n_{k}+v_{k} .
$$

The quantization noise $v_{k}=v_{k}\left(x_{k}, m_{k}\right)$ of variance ${ }^{3}$ [19]

$$
q_{k}^{2}=\frac{M^{2}}{12\left(2^{m_{k}}-1\right)^{2}},
$$

can be shown to be white (over sensors) and independent of the input signal $r_{k}=x_{k}+n_{k}$, with $M$ standing for the dynamic range of the spatial field (namely, $x_{k} \in[-M, M]$ ). Since the uniform quantizer is unbiased, we have $\mathbb{E}\left\{y_{k}\right\}=x_{k}$ and $\operatorname{Var}\left\{y_{k}\right\}=\sigma_{k}^{2}$. Further, it can be shown that $\sigma_{k}^{2}=q_{k}^{2}+\eta_{k}^{2}$.

\footnotetext{
${ }^{3}$ This holds true for a wider class of input signals and some conditions on the dithering signal (see [19] for details).
}

The $K$ nodes in the sensor network convey their measurements to the FC over a set of $K$ orthogonal channels. Assuming that each sensor samples the spatial field at a rate of $R_{\mathrm{S}}$ samples/s, a reliable transmission occurs when the encoding rate $R_{\mathrm{s}} m_{k}$ satisfies

$$
R_{s} m_{k} \leq w_{k} \log _{2}\left(1+\frac{p_{k}\left|h_{k}\right|^{2}}{w_{k} \mathrm{~N}_{\mathrm{o}}}\right) \quad[\mathrm{b} / \mathrm{s}]
$$

where $w_{k}$ and $p_{k}$ stand for the bandwidth and transmit power allocated to the $k$-th channel/sensor, and $\mathrm{N}_{\mathrm{o}}$ denotes the spectral density of the noise in the sensor-to-FC channels. Clearly, the statistics of the squared gains $\gamma_{k} \triangleq\left|h_{k}\right|^{2}, k=1, \ldots, K$, depend on the particular channel model under consideration (e.g., Gaussian, Rayleigh-fading). Besides, we impose a total bandwidth constraint $W$ and a sum-power constraint $P$ and we evenly allocate power and bandwidth ${ }^{4}$ to the set of $K$ sensors, this yielding $p_{k}=P / K$ and $w_{k}=W / K$. For the ease of notation and without loss of generality, we impose $R_{s}=1$ and, hence, the following inequality holds for the number of bits per sample at the output of the $k$-th quantizer

$$
m_{k} \leq \frac{W}{K} \log _{2}\left(1+\mathrm{SNR} \cdot \gamma_{k}\right),
$$

with SNR $\triangleq \frac{P}{W N_{\circ}}$ denoting the average SNR in the sensor-toFC channels.

\section{ReCONSTRUCtion of the Spatial Field: DISTORTION ANALYSIS}

Our goal is to reconstruct the spatial field $x(\mathbf{s})$ for all $\mathbf{s} \in \mathcal{S}$ from the set of samples $\left\{y_{k}\right\}$ collected at the FC. As a result of the spatial sampling process and the channel bandwidth constraint, the reconstructed field $\hat{X}(\mathbf{s})$ is unavoidably subject to some distortion. In the sequel, such distortion will be characterized by the average normalized mean square error (MSE), namely,

$$
\operatorname{MSE}=\frac{1}{E_{x}} \mathbb{E}\left\{\int_{\Re^{l}}(\hat{X}(\mathbf{s})-x(\mathbf{s}))^{2} d \mathbf{s}\right\}
$$

where the expectation in taken over the realizations of the spatial sampling process, the observation and the quantization noise. In order to define an appropriate reconstruction strategy, first we need to analyze the spectrum of the received signal. The sampled and quantized version of the spatial field $Y(\mathbf{s})$ which is available at the FC can be expressed as

$$
Y(\mathbf{s})=\sum_{k} y_{k} \delta\left(\mathbf{s}-\mathbf{s}_{k}\right)=X_{s}(\mathbf{s})+W(\mathbf{s}),
$$

where $W(\mathbf{s}) \triangleq \sum_{k} w_{k} \delta\left(\mathbf{s}-\mathbf{s}_{k}\right)$ with $w_{k}=n_{k}+v_{k}$ accounting for the combined effects of the observation and quantization noise. Let us now define the average (over $\mathbf{s}$ ) statistical autocorrelation function $\bar{R}_{Y}(\boldsymbol{\tau})$ and spectral density

\footnotetext{
${ }^{4}$ This is consistent with the fact that, in Gaussian channels (see Section IV-A ahead), the sensor-to-FC channels are identical and, in Rayleigh-fading channels (Section IV-B), sensor operate without transmit CSI. In scenarios with transmit CSI, power and/or bandwidth allocation schemes typically result into substantial performance gains (see e.g. [8], [20], [21]).
} 


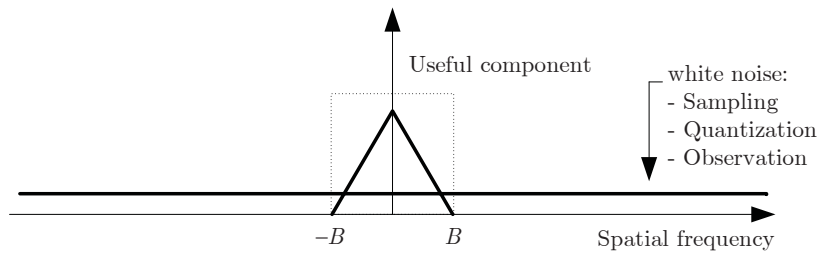

Fig. 2. Diagram of the spectral components of the sampled spatial field. The one-dimensional case is considered here for the sake of illustration.

$\overline{\mathcal{E}}_{Y}(\boldsymbol{\nu})$ of $Y(\mathbf{s})$ as

$$
\begin{aligned}
\bar{R}_{Y}(\boldsymbol{\tau}) & =\int_{\Re^{l}} \mathbb{E}\{Y(\mathbf{s}) Y(\mathbf{s}-\boldsymbol{\tau})\} d \mathbf{s} \\
& =\bar{R}_{X_{s}}(\boldsymbol{\tau})+\bar{R}_{W}(\boldsymbol{\tau}) \\
\overline{\mathcal{E}}_{Y}(\boldsymbol{\nu}) & =\mathfrak{F}^{(l)}\left[\bar{R}_{Y}(\boldsymbol{\tau})\right],
\end{aligned}
$$

where $\bar{R}_{X_{s}}(\boldsymbol{\tau})$ and $\bar{R}_{W}(\boldsymbol{\tau})$ denote the average autocorrelation function of $X_{s}(\mathbf{s})$ and $W(\mathbf{s})$, respectively. Clearly, $\bar{R}_{X_{s}}(\boldsymbol{\tau})$ can be re-written as

$$
\begin{aligned}
\bar{R}_{X_{s}}(\boldsymbol{\tau}) & =\int_{\Re^{l}} \mathbb{E}\{x(\mathbf{s}) x(\mathbf{s}-\boldsymbol{\tau}) P(\mathbf{s}) P(\mathbf{s}-\boldsymbol{\tau})\} d \mathbf{s} \\
& =\int_{\Re^{l}} x(\mathbf{s}) x(\mathbf{s}-\boldsymbol{\tau}) R_{P}(\boldsymbol{\tau}) d \mathbf{s}=R_{x}(\boldsymbol{\tau}) \cdot R_{P}(\boldsymbol{\tau}),
\end{aligned}
$$

and, hence, the corresponding energy spectral density reads

$$
\overline{\mathcal{E}}_{X_{s}}(\boldsymbol{\nu})=\mathfrak{F}^{(l)}\left[\bar{R}_{X_{s}}(\boldsymbol{\tau})\right]=\mathcal{E}_{x}(\boldsymbol{\nu}) \otimes S_{P}(\boldsymbol{\nu}) .
$$

From (10) and (22), it follows that

$$
\overline{\mathcal{E}}_{X_{s}}(\boldsymbol{\nu})=\rho^{2} \mathcal{E}_{x}(\boldsymbol{\nu})+E_{x} \cdot \rho .
$$

Finally, the average energy spectral density of $Y(\mathbf{s})$ can be expressed as

$$
\begin{aligned}
\overline{\mathcal{E}}_{Y}(\boldsymbol{\nu}) & =\overline{\mathcal{E}}_{X_{s}}(\boldsymbol{\nu})+\overline{\mathcal{E}}_{W}(\boldsymbol{\nu}) \\
& =\rho^{2} \mathcal{E}_{x}(\boldsymbol{\nu})+E_{x} \cdot \rho+\overline{\mathcal{E}}_{W}(\boldsymbol{\nu}),
\end{aligned}
$$

where $\overline{\mathcal{E}}_{W}(\boldsymbol{\nu})=\mathfrak{F}^{(l)}\left[\bar{R}_{W}(\boldsymbol{\tau})\right]$. From (24), one notices that the spectrum of the sampled signal is composed of three terms (see Fig. 2). The first one, the useful component, is proportional to the spectrum of the spatial field $x(\mathbf{s})$. The second term captures the effect of the white sampling noise associated to the random nature of the sampling process. The last term accounts for both quantization and observation noises. From Fig. 2, it becomes apparent that the spatial field can be reconstructed from its samples by means of an ideal low-pass filter ${ }^{5}$ that separates the useful component from outof-band noise. This is equivalent to the interpolation of the received set of samples $Y(\mathbf{s})$. The estimate $\hat{X}(\mathbf{s})$ of the spatial field can thus be expressed as

$$
\hat{X}(\mathbf{s})=Y(\mathbf{s}) \otimes \phi(\mathbf{s})=\sum_{k} x_{k} \phi\left(\mathbf{s}-\mathbf{s}_{k}\right)+\sum_{k} w_{k} \phi\left(\mathbf{s}-\mathbf{s}_{k}\right),
$$

\footnotetext{
${ }^{5}$ In general, this estimator is not optimal (i.e. in a MSE sense) but, it is the best one can do in the absence of any prior information about the spectrum of $X(s)$
}

where $\phi(\mathbf{s})$ is the impulse response of the linear interpolator. For an ideal low-pass filter, its transfer function $\Phi(\boldsymbol{\nu})=$ $\mathfrak{F}^{(l)}[\phi(\mathbf{s})]$ is given by

$$
\Phi(\boldsymbol{\nu})=\left\{\begin{array}{lr}
1 / \rho & \boldsymbol{\nu} \in \mathcal{S} \\
0 & \text { otherwise }
\end{array}\right.
$$

where, in the above expression, the normalization factor $1 / \rho$ in (26) has been set conventionally in order to have a useful component with energy $E_{x}$ at the output. For notational convenience, in the sequel we attempt to express the normalized estimation error in the reconstruction of the whole spatial field of (17) as a function of the estimation error in the sampled locations. Conditioned on signal $x(\mathbf{s})$, the $y_{k}$ samples are independent and, hence, the best linear estimate of $x_{k}$ turns out to be $\hat{x}_{k}=y_{k}$, which is unbiased. The distortion in the estimate of sample $x_{k}$ is thus given by

$$
\begin{aligned}
D_{k} & =\frac{1}{E_{x}} \mathbb{E}\left\{\left(\hat{x}_{k}-x_{k}\right)^{2}\right\}=\frac{\sigma_{k}^{2}}{E_{x}} \\
& =\frac{1}{\Gamma_{\mathrm{obs}, k}}+\frac{q_{k}^{2}}{E_{x}}=\frac{1}{\Gamma_{\mathrm{obs}, k}}+\frac{F}{\left(2^{m_{k}}-1\right)^{2}}
\end{aligned}
$$

with $F=\frac{M^{2}}{12 E_{\gamma_{5}}}$ being proportional to peak factor of the spatial field $F_{p}=\frac{M^{2}}{E_{x}}$. The normalized distortion $\bar{D}$ averaged over the realizations of the sampling process yields

$$
\bar{D}=\mathbb{E}_{K}\left\{\sum_{k=1}^{K} D_{k}\right\}=\sum_{K=0}^{\infty} p_{K}(K) \sum_{k=1}^{K} D_{k}
$$

with $p_{K}(K)$ given by $(11)^{6}$. Finally, and after some rearrangements shown in the Appendix, the normalized estimation error of (17) can be conveniently expressed as

$$
\operatorname{MSE}=\frac{\beta}{\rho}\left(1+\frac{1}{\rho} \bar{D}\right)
$$

where to recall, $\beta$ denotes the Lebesgue measure of $\mathcal{S}$, namely, $\beta=(2 B)^{l}$.

\section{Optimal Network Design}

Here, we particularize the aforementioned distortion analysis for Gaussian and Rayleigh-fading scenarios. On that basis, we then derive closed-form expressions of the optimal sensor density and, where applicable, the optimal encoding rate.

\section{A. Gaussian Channels}

In Gaussian channels, the channel (squared) gains read $\gamma_{k}=$ $1 \forall k$. Further, we assume identical observation SNRs in the sensor nodes $\left(\Gamma_{\mathrm{obs}, k}=\Gamma_{\mathrm{obs}}\right)$. Hence, from (27) and (28) and by taking (16) with equality, it turns out that

$$
\bar{D}=\frac{\rho A}{\Gamma_{\mathrm{obs}}}+\mathbb{E}_{K}\left\{\frac{K F}{\left((1+\mathrm{SNR})^{\frac{W}{K}}-1\right)^{2}}\right\}
$$

Unfortunately, a closed form expression of $\bar{D}$ is extremely difficult to obtain and, hence, the actual MSE cannot be used as a score function to optimize on $\rho$. However, the argument

\footnotetext{
${ }^{6}$ This follows from the fact that, clearly, the overall MSE in the samples does not depend on their actual locations but on its number $K$.
} 
in the expectation function is convex in $K$ and, thus, we can resort to the following lower bound:

$$
\bar{D} \geq \frac{\rho A}{\Gamma_{\text {obs }}}+\frac{\rho A F}{\left((1+\mathrm{SNR})^{\frac{W}{\rho A}}-1\right)^{2}}
$$

which follows from Jensen's inequality. Now, by replacing (31) into (29) it yields

$$
\begin{aligned}
\mathrm{MSE} & =\frac{\beta}{\rho}\left(1+\frac{1}{\rho} \bar{D}\right) \\
& \geq \frac{\beta}{\rho}\left(1+\frac{A}{\Gamma_{\mathrm{obs}}}+\frac{A F}{\left((1+\mathrm{SNR})^{\frac{W}{\rho A}}-1\right)^{2}}\right) \\
& \geq \frac{\beta}{\rho}\left(1+\frac{A}{\Gamma_{\mathrm{obs}}}+\frac{A F}{(1+\mathrm{SNR})^{2 \frac{W}{\rho A}}}\right) .
\end{aligned}
$$

The first two terms in (33) account for the contribution of the sampling noise and the (filtered) observation noise to the normalized MSE. Both are monotonically decreasing functions of the node density $\rho$. In other words, having a denser grid of sensor is, in principle, beneficial in terms of accuracy in the reconstructed spatial field ${ }^{7}$. The third term in the summation accounts for the impact of the quantization noise. Intuitively, it is a monotonically increasing function in $\rho$ : under a total bandwidth constraint, when the node density increases the available rate per sensor diminishes, this resulting into a rougher quantization of the sensor observations (this can also be formally proved). From this discussion, it follows that there exists an optimal operating point $\rho^{*}$ for which the MSE in the reconstructed spatial field is minimized. By assuming that $(1+\mathrm{SNR})^{\frac{W}{\rho A}} \gg 1$, we can lower-bound (33) by (34). By solving the corresponding minimization problem, namely,

$$
\rho^{*} \approx \arg \min _{\rho} \frac{\beta}{\rho}\left(1+\frac{A}{\Gamma_{\text {obs }}}+\frac{A F}{(1+\mathrm{SNR})^{2 \frac{W}{\rho A}}}\right),
$$

an approximate yet in general accurate expression of the optimal node density can be obtained (see numerical results in Section V). More precisely, by setting the first derivative of the lower bound in (34) to zero, we have that

$$
\rho^{*} \approx \frac{2 W \ln (1+\mathrm{SNR})}{A\left(1-\mathrm{W}_{-1}\left(-\frac{A+\Gamma_{\mathrm{obs}}}{A \Gamma_{\mathrm{obs}} F}\right)\right)}
$$

where $W_{-1}(\cdot)$ stands for the negative real branch of the Lambert function [22]. It is worth noting that the solution of (36) is only feasible in the real domain of $\mathrm{W}_{-1}(\cdot)$ that is, for $\frac{A+\Gamma_{\mathrm{obs}}}{A \Gamma_{\mathrm{obs}} T} \leq \frac{1}{e}$.

Finally, from (34) and (36) and by letting the overall rate $W \ln (1+\mathrm{SNR})$ grow without bound, we realize that the scaling law of the lower bound reads

$$
\mathrm{MSE} \sim \frac{C}{W \ln (1+\mathrm{SNR})}
$$

with $C$ standing for a positive constant. Furthermore, computer simulation results in Section $\mathrm{V}$ ahead show that the scaling law of the actual MSE is identical to that of its lower bound.

\footnotetext{
${ }^{7}$ This follows from the fact that the component associated to the useful signal in the spectral density of $(24)$ is proportional to $\rho^{2}$ whereas e.g. the sampling noise term scales with $\rho$.
}

\section{B. Rayleigh-fading Channels}

Now, the squared channel gains $\gamma_{k} \forall k$ turn out to be i.i.d. random variables with unit-mean exponential distribution. In realistic scenarios, sensors cannot acquire and track instantaneous transmit CSI and, hence, the bit rate cannot be dynamically adjusted to match the actual channel conditions. For this reason, we hereinafter assume that each sensor observation is quantized at a common and constant (i.e. time-invariant) bit rate $R$. This unavoidably entails some outage probability $p_{\text {out }}$, namely, the probability that the source rate $R$ exceeds the instantaneous channel rate:

$$
\begin{aligned}
p_{\text {out }} & =\operatorname{Pr}\left(\frac{W}{K} \log _{2}\left(1+\mathrm{SNR} \cdot \gamma_{k}\right)<R\right) \\
& =F_{\gamma}\left(\frac{2^{K R / W}-1}{\mathrm{SNR}}\right)
\end{aligned}
$$

where $F_{\gamma}(\cdot)$ denotes the cumulative density function of the random variable $\gamma$ (i.e., $F_{\gamma}(x)=1-e^{-x}$ for Rayleighfading channels). The aggregated rate for the whole WSN reads $R_{\text {aggr }}=K R$ and it can be expressed as a function of the outage probability as follows:

$$
R_{\mathrm{aggr}}=W \log _{2}\left(1-\mathrm{SNR} \ln \left(1-p_{\text {out }}\right)\right) .
$$

The first step towards the derivation of the optimal aggregated rate $R_{\mathrm{aggr}}^{*}$ and its associated optimal average node density $\rho^{*}$ is to identify the impact of the outage probability on the sampling pattern. Let $K_{s}$ be the actual number of successfully (i.e. not in outage) decoded samples at the FC. Since outage events are independent over sensors (because so are channel gains), $K_{s}$ turns out to be a Poisson r.v. with equivalent spatial density given by $\rho\left(1-p_{\text {out }}\right)$. From (29), the normalized MSE yields:

$$
\operatorname{MSE}=\frac{\beta}{\rho\left(1-p_{\text {out }}\right)}\left(1+\frac{1}{\rho\left(1-p_{\text {out }}\right)} \bar{D}\right),
$$

where $\bar{D}$ is now given by

$$
\bar{D}=\frac{\rho\left(1-p_{\text {out }}\right) A}{\Gamma_{\text {obs }}}+\mathbb{E}_{K_{s}, K}\left\{\frac{K_{s} F}{\left(2^{\frac{R_{\text {aggr }}}{K}}-1\right)^{2}}\right\}
$$

Again, by resorting to Jensen's inequality we have that

$$
\begin{aligned}
\bar{D} & =\frac{\rho\left(1-p_{\text {out }}\right) A}{\Gamma_{\text {obs }}}+\mathbb{E}_{K_{s}, K}\left\{\frac{K_{s} F}{\left(2^{\frac{R_{\text {aggr }}}{K}}-1\right)^{2}}\right\} \\
& =\frac{\rho\left(1-p_{\text {out }}\right) A}{\Gamma_{\text {obs }}}+\mathbb{E}_{K}\left\{\frac{\mathbb{E}_{K_{s} \mid K}\left\{K_{s} \mid K\right\} F}{\left(2^{\frac{R_{\text {aggr }}}{K}}-1\right)^{2}}\right\} \\
& =\frac{\rho\left(1-p_{\text {out }}\right) A}{\Gamma_{\text {obs }}}+\mathbb{E}_{K}\left\{\frac{\left(1-p_{\text {out }}\right) K F}{\left(2^{\frac{R_{\text {aggr }}}{K}}-1\right)^{2}}\right\} \\
& \geq \frac{\rho\left(1-p_{\text {out }}\right) A}{\Gamma_{\text {obs }}}+\frac{\rho\left(1-p_{\text {out }}\right) A F}{\left(2^{\frac{R_{\text {aggr }}}{\rho A}}-1\right)^{2}} \\
& \geq \frac{\rho\left(1-p_{\text {out }}\right) A}{\Gamma_{\text {obs }}}+\frac{\rho\left(1-p_{\text {out }}\right) A F}{2^{2 \frac{R_{\text {aggr }}}{\rho A}}}
\end{aligned}
$$


where (45) follows from the fact that $K_{s} \mid K$ is a binomial random variable with probability of activation $1-p_{\text {out }}$ and population size $K$. In the last inequality, we have assumed once again that $2^{\frac{R_{\text {aggr }}}{\rho A}} \gg 1$. By replacing the right handside of (47) into (41), a lower bound of the MSE follows

$$
\operatorname{MSE} \geq \operatorname{MSE}_{\mathrm{LB}}=\frac{\beta}{\rho\left(1-p_{\text {out }}\right)}\left(1+\frac{A}{\Gamma_{\text {obs }}}+\frac{A F}{2^{2 \frac{R_{\text {aggr }}}{\rho A}}}\right)
$$

which will be used to derive (tight) approximations of the optimal aggregated rate, $R_{\mathrm{aggr}}^{*}$, and the optimal spatial density, $\rho^{*}$. From (40), $R_{\mathrm{aggr}}^{*}$ is, in turn, a function of $\rho$ and $p_{\text {out }}$ and, therefore, we equivalently minimize with respect to these two variables. The optimization problem can be re-written as follows [23]:

$$
\begin{aligned}
\min _{\rho, p_{\text {out }}} \operatorname{MSE}_{\mathrm{LB}}\left(\rho, p_{\text {out }}\right) & =\min _{p_{\text {out }}}\left(\min _{\rho} \operatorname{MSE}_{\mathrm{LB}}\left(\rho, p_{\text {out }}\right)\right) \\
& \triangleq \min _{p_{\text {out }}} \operatorname{MSE}_{\mathrm{LB}}\left(p_{\text {out }}\right)
\end{aligned}
$$

The solution to the inner minimization problem in (49) yields:

$$
\rho^{*}\left(p_{\text {out }}\right)=\frac{2 W \ln \left(1-\mathrm{SNR} \ln \left(1-p_{\text {out }}\right)\right)}{A\left(1-\mathrm{W}_{-1}\left(-\frac{A+\Gamma_{\text {obs }} e}{A \Gamma_{\text {obs }} F}\right)\right)} .
$$

By inserting (51) into the outer optimization problem (50) and then setting its first derivative to zero, the optimal outage probability follows:

$$
p_{\text {out }}^{*}=1-e^{\frac{1}{\text { SNR }}-\frac{1}{\mathrm{w}_{0}(\mathrm{SNR})}} .
$$

with $W_{0}(\cdot)$ denoting the principal branch of the Lambert function [22]. Finally, from (40), (51), and (52) the optimal encoding rate and node density read

$$
\begin{aligned}
R^{*} & \approx \frac{R_{\mathrm{aggr}}^{*}}{K} \\
& =\frac{W}{2 K \ln (2)}\left(1-\mathrm{W}_{-1}\left(-\frac{A+\Gamma_{\mathrm{obs}}}{A \Gamma_{\mathrm{obs}} F} e\right)\right) \\
\rho^{*} & \approx \frac{2 W \cdot \mathrm{W}_{o}(\mathrm{SNR})}{A\left(1-\mathrm{W}_{-1}\left(-\frac{A+\Gamma_{\mathrm{obs}}}{A \Gamma_{\mathrm{obs}} F}\right)\right)} .
\end{aligned}
$$

Again, by letting the SNR grow without bound the scaling law of the lower bound of the actual MSE follows, namely,

$$
\mathrm{MSE} \sim \frac{B}{W \cdot \mathrm{W}_{o}(\mathrm{SNR})}
$$

with $B$ standing for a positive constant. Finally, from [22] we know that

$$
\lim _{\mathrm{SNR} \rightarrow \infty} \frac{\ln (\mathrm{SNR})}{\mathrm{W}_{o}(\mathrm{SNR})}=1
$$

and, thus, the scaling law is identical to that of Gaussian channels.

\section{NumericAl RESUltS}

In this section, we present some numerical results aimed to validate the proposed methodology for the optimal dimensioning of WSNs. Details on specific parameter settings can be found in the figure captions.

To start with, we focus our attention in scenarios with Gaussian channels. First, in Fig. 3, we depict the actual MSE and

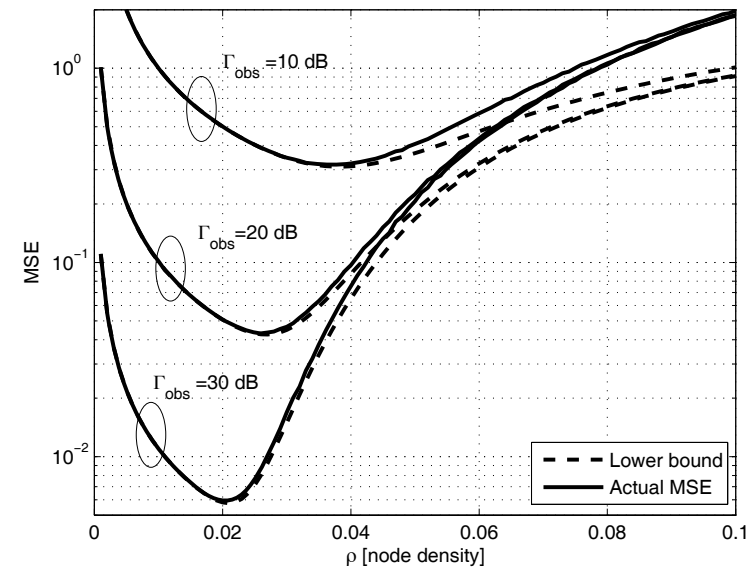

Fig. 3. Normalized MSE as a function of the spatial density $\rho$ ( $W=500$ $\left.\mathrm{kHz}, R_{s}=1 \mathrm{kHz}, F=10, \mathrm{SNR}=10 \mathrm{~dB}, \beta=1 e-5 \mathrm{~m}^{-2}\right)$.

the lower bound given by (34) as a function of the (average) sensor density $\rho$. Curves are parameterized for several values of the observation signal-to-noise ratio, $\Gamma_{\text {obs }}$. As anticipated, there exists an optimal operating point on each curve where the best trade-off in terms of sampling, observation and quantization noise is attained. Clearly, by diminishing the observation SNR the optimal density of sensors increases, as suggested by (36). This means that the spatial field is optimally reconstructed if the extra observation noise is averaged out over a larger number of spatial samples, even if this entails some increase in the quantization noise. Still, this cannot avoid a severe degradation in the normalized MSE: $6 \cdot 10^{-3}$ for $\Gamma_{\text {obs }}=30 \mathrm{~dB}$ vs. $3 \cdot 10^{-2}$ for $\Gamma_{\text {obs }}=10 \mathrm{~dB}$. However, the optimal node density is not a function of the spatial bandwidth of the field: from (33), the only impact of the per-dimension spatial bandwidth $B_{z}$ of the field (or, in other words, its spatial variability) is in terms of a scale factor in the normalized $\mathrm{MSE}^{8}$. This somewhat counter-intuitive result (one could e.g. think that fields with higher spatial variability require a denser grid of sensors) follows from the fact that the spatial field is randomly sampled. Being the inter-sensor distance a random variable, the optimally designed sensor density turns out to be optimal regardless of the bandwidth of the spatial field. This is in stark contrast with our previous works with deterministic (i.e. uniform) sampling patterns, where the optimal number of nodes depends on the spatial variability of the field. Finally, we also observe that, overall, the lower bound of the MSE (34) is tight. This is particularly true in the region around the optimal operating point and, hence, the performance loss stemming from the use of the approximate node density (36) is negligible. On the contrary, the gap between both curves is wider for increasing values of $\rho$ since, in this region, the assumption $(1+\mathrm{SNR})^{\frac{W}{\rho A}} \gg 1$ does not hold.

Figure 4 provides further insights on the impact of a number of system parameters on the optimal node density. As discussed above, $\rho^{*}$ is a decreasing function in the observation SNR. Besides, increasing $W$ results into higher values of the

\footnotetext{
${ }^{8}$ Since the amount of noise at the output of the interpolation filter $\Phi(\boldsymbol{\nu})$ is proportional to $\beta=B_{z}^{l}$.
} 


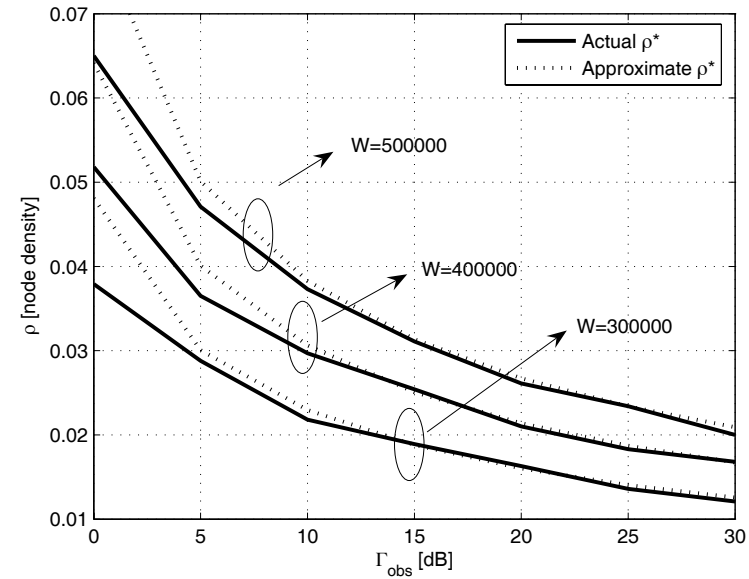

Fig. 4. Optimal sensor density as a function of the observation SNR for different values of $W$.

optimal node density since, clearly, more bandwidth per sensor is made available. Interestingly, the approximation turns out to be particularly accurate in the high- $\Gamma_{\text {obs }}$ region. This stems from the fact that, for this range of values, the associated $\rho^{*}$ values are smaller which, again, is beneficial for the approximation $(1+\mathrm{SNR})^{\frac{W}{\rho A}} \gg 1$.

Next, we present some results for scenarios with Rayleighfading channels. Figure 5 shows the contour plot of the normalized MSE as a function of the node density and outage probability given by (48). Clearly, the function depicted in Fig. 5 has single global minimum. Complementarily, Fig.6.a shows a horizontal section of the previous plot. More precisely, it depicts the MSE evaluated at the optimal $\rho$ (computed numerically) along with the corresponding lower bound (51) as a function of the outage probability. Again, the lower bound is tight for the whole range of values and, hence, the optimal outage probability can be accurately computed in a closed-form from (52). Likewise, the lower bound of the MSE evaluated at the optimal $p_{\text {out }}$ is also tight (see Fig. 6.b), this meaning that the problem (49) can also be accurately solved if the order in which the inner and outer problems are solved is reversed.

Finally, Figure 7 depicts the scaling law for Gaussian and Rayleigh-fading channels without transmit CSI. Several conclusions are in line. First of all, we observe that, in both scenarios, the lower bound and the corresponding actual MSE curves exhibit the same scaling law (and, furthermore, the lower bound is very tight). Besides, it is also clear that the scaling laws for the Gaussian and Rayleigh-fading channels are identical. However, there is a gap between the corresponding scaling laws due to the fact that, in the absence of instantaneous transmit CSI, fading has a negative impact in terms of reconstruction MSE.

\section{CONCLUSIONS}

In this paper, we have addressed the problem of the estimation of bandlimited two-dimensional spatial fields under a total bandwidth constraint in the sensor-to-FC channels. Besides, we have also assumed that sensor nodes are randomly deployed according to a homogeneous (spatial) Poisson

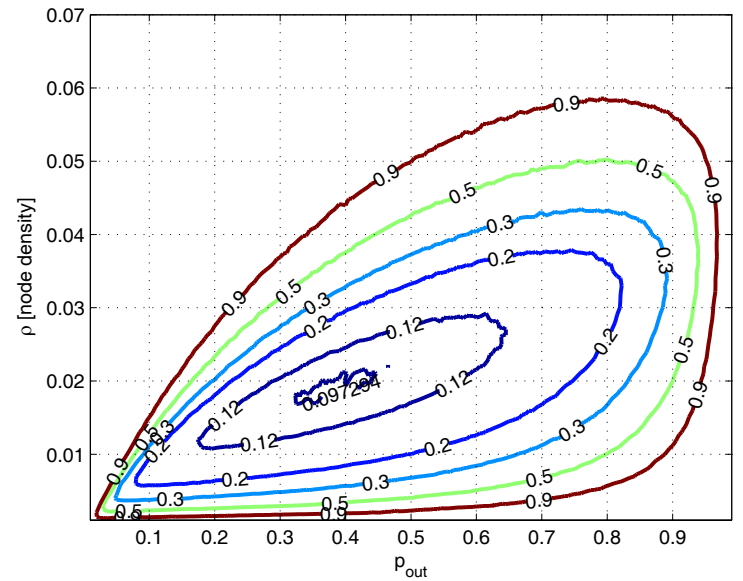

Fig. 5. Contour plot of the normalized MSE as a function of the node density and outage probability $\left(\Gamma_{\text {obs }}=20 \mathrm{~dB}, W=500 \mathrm{kHz}, R_{s}=1\right.$ $\mathrm{kHz}, F=10, \mathrm{SNR}=10 \mathrm{~dB}, \beta=1 e-5 \mathrm{~m}^{-2}$ ).

Point Process. In this context, we have found an analytical expression of the MSE in the reconstructed spatial field which includes the contributions of the observation, quantization and sampling noises. This has been done for two scenarios of interest: Gaussian channels, on the one hand, and Rayleighfading channels without transmit CSI, on the other. On the basis of a lower bound of the MSE, we have derived an approximate closed-form expression of the optimal sensor density which attains the best trade-off for the aforementioned noise contributions. Besides, for the Rayleigh-fading scenario, we have analytically derived an expression of the rate at which the observations must be encoded (i.e. quantized). We have also found that the MSE (and its lower bound) in the highSNR regime scale as $\frac{1}{\ln (\mathrm{SNR})}$ and $\frac{1}{\mathrm{~W}_{o}(\mathrm{SNR})}$ in Gaussian and Rayleigh-fading scenarios, respectively. Computer simulation results reveal that, for Gaussian channels, the lower bound is in general tight and, thus, the approximation of the optimal node density is accurate. Interestingly, the optimal operating point depends on a number of system parameters (e.g. total bandwidth, variance of the observation noise, SNR in the sensor-to-FC channels) but not on the spatial bandwidth of the field, this being a consequence of the random sampling pattern. In Rayleigh-fading scenarios, the approximations of the node density and the optimal outage probability (or, equivalently, the aggregated encoding rate) are very accurate, as well. Finally, we have also found that, although the scaling laws of the MSE in Gaussian and Rayleigh-fading channels are identical, there exists a gap motivated by the fact that, in the absence of instantaneous transmit CSI, fading always has a negative impact.

\section{APPENDIX}

By expanding the definition (17), we can write

$$
\begin{aligned}
\mathrm{MSE} & =\frac{1}{E_{x}}\left[\mathbb{E}\left\{\int_{\Re^{l}} \hat{X}^{2}(\mathbf{s}) d \mathbf{s}\right\}+\int_{\Re^{l}} x^{2}(\mathbf{s}) d \mathbf{s}\right. \\
& \left.-2 \mathbb{E}\left\{\int_{\Re^{l}} \hat{X}(\mathbf{s}) \cdot x(\mathbf{s}) d \mathbf{s}\right\}\right] .
\end{aligned}
$$



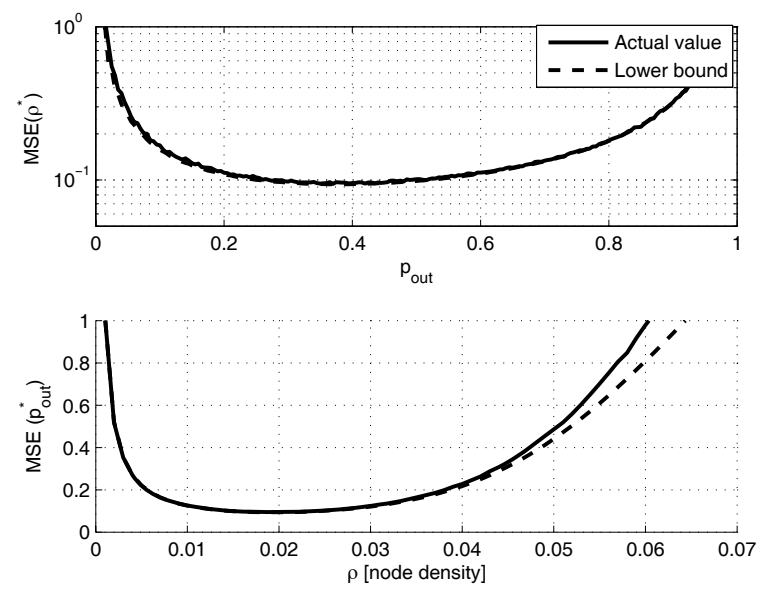

Fig. 6. a) MSE evaluated at the optimal $\rho^{*}$ as a function of the outage probability. b) MSE evaluated at the optimal $p_{\text {out }}^{*}$ as a function of the sensor density. ( $\Gamma_{\text {obs }}=20 \mathrm{~dB}, W=500 \mathrm{kHz}, R_{s}=1 \mathrm{kHz}, F=10$, SNR $=10$ $\left.\mathrm{dB}, \beta=1 e-5 \mathrm{~m}^{-2}\right)$.

From Parseval's relationship and taking into account that $\int_{\Re^{l}} E_{X}(\boldsymbol{\nu}) d \nu=E_{x}$, the first term in (56) reads

$$
\begin{aligned}
\frac{1}{E_{x}} \int_{\Re^{l}} \mathbb{E}\left\{\hat{X}^{2}(\mathbf{s})\right\} d \mathbf{s} \\
=\frac{1}{E_{x}} \int_{\Re^{l}}|\Phi(\boldsymbol{\nu})|^{2} \mathfrak{F}^{(l)}[\mathbb{E}\{Y(\mathbf{s}) Y(\mathbf{s}-\boldsymbol{\tau})\}] d \boldsymbol{\nu} \\
=\frac{1}{E_{x}} \int_{\Re^{l}}|\Phi(\boldsymbol{\nu})|^{2} \overline{\mathcal{E}}_{X_{s}}(\boldsymbol{\nu}) d \boldsymbol{\nu} \\
\quad+\frac{1}{E_{x}} \int_{\Re^{l}}|\Phi(\boldsymbol{\nu})|^{2} \overline{\mathcal{E}}_{W}(\boldsymbol{\nu}) d \boldsymbol{\nu} .
\end{aligned}
$$

Now the autocorrelation function of the process $W(\mathbf{s})$ can be expressed as

$$
\begin{aligned}
\bar{R}_{W}(\boldsymbol{\tau}) & =\int_{\Re^{l}} \mathbb{E}\{W(\mathbf{s}) W(\mathbf{s}-\boldsymbol{\tau})\} d \mathbf{s} \\
& =\mathbb{E}\left\{\sum_{k=1}^{K} \sum_{h=1}^{K} w_{k} w_{h} \int_{\Re^{l}} \delta\left(\mathbf{s}-\mathbf{s}_{k}\right) \delta\left(\mathbf{s}-\boldsymbol{\tau}-\mathbf{s}_{h}\right) d \mathbf{s}\right\} \\
& =\delta(\boldsymbol{\tau}) \mathbb{E}\left\{\sum_{k=1}^{K} \sigma_{k}^{2}\right\} .
\end{aligned}
$$

where we have used the property that $w_{k}$ 's are independent and the last expectation is with respect to the r.v. $K$. Hence, the energy spectral density is

$$
\overline{\mathcal{E}}_{W}(\boldsymbol{\nu})=\mathfrak{F}^{(l)}\left[\bar{R}_{W}(\boldsymbol{\tau})\right]=\mathbb{E}\left\{\sum_{k=1}^{K} \sigma_{k}^{2}\right\} .
$$

By substituting (59) into (57) we get

$$
\begin{aligned}
\frac{1}{E_{x}} \int_{\Re^{l}} \mathbb{E}\left\{\hat{X}^{2}(\mathbf{s})\right\} d \mathbf{s} \\
=1+\frac{\beta}{\rho}+\frac{1}{E_{x}} \mathbb{E}\left\{\sum_{k=1}^{K} \sigma_{k}^{2}\right\} \int_{\Re^{l}}|\Phi(\boldsymbol{\nu})|^{2} d \boldsymbol{\nu} \\
=1+\frac{\beta}{\rho}+\frac{\beta}{\rho^{2}} \mathbb{E}\left\{\sum_{k} \frac{\sigma_{k}^{2}}{E_{x}}\right\}
\end{aligned}
$$

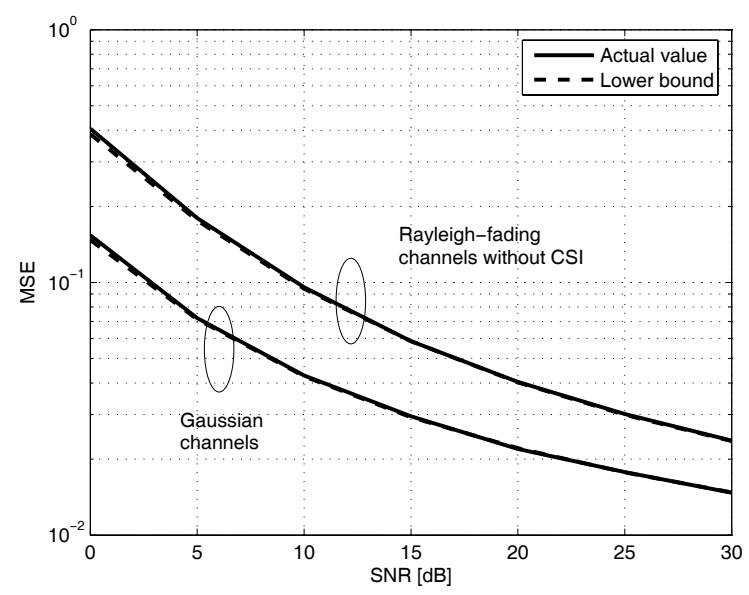

Fig. 7. Normalized MSE as a function of the SNR in the sensor-to-FC channels $\left(\Gamma_{\text {obs }}=20 \mathrm{~dB}, W=500 \mathrm{kHz}, R_{s}=1 \mathrm{kHz}, F=10, \beta=1 e-5\right.$ $\mathrm{m}^{-2}$ ).

$$
=1+\frac{\beta}{\rho}+\frac{\beta}{\rho^{2}} \bar{D},
$$

where we recalled the definition of $\bar{D}$ in (28). The second term is equal to 1 whereas the third term is given by

$$
\begin{aligned}
& -\frac{2}{E_{x}} \int_{\Re^{l}} \mathbb{E}\{\hat{X}(\mathbf{s})\} \cdot x(\mathbf{s}) d \mathbf{s} \\
& \quad=-\frac{2}{E_{x}} \int_{\Re^{l}} \Phi(\boldsymbol{\nu}) \mathbb{E}\left\{S_{Y}(\boldsymbol{\nu})\right\} S_{x}^{*}(\boldsymbol{\nu}) d \boldsymbol{\nu} .
\end{aligned}
$$

where $S_{Y}(\boldsymbol{\nu})=\mathfrak{F}^{(l)}[Y(\mathbf{s})]$. Due to the stationarity of the random process $P(\mathbf{s})$ and considering that $\mathbb{E}\left\{w_{k}\right\}=0$, it is

$$
\mathbb{E}\left\{S_{Y}(\boldsymbol{\nu})\right\}=\mathbb{E}\left\{\mathfrak{F}^{(l)}[x(\mathbf{s}) \cdot P(\mathbf{s})]\right\}=\mu_{P} \cdot S_{x}(\boldsymbol{\nu}) .
$$

By substitution of (62), (61) becomes

$$
\begin{array}{rl}
-\frac{2}{E_{x}} \int_{\Re^{l}} & \mathbb{E}\{\hat{X}(\mathbf{s})\} \cdot x(\mathbf{s}) d \mathbf{s} \\
& =-\frac{2}{E_{x}} \rho \int_{\Re^{l}} \Phi(\boldsymbol{\nu}) \overline{\mathcal{E}}_{X}(\boldsymbol{\nu}) d \boldsymbol{\nu}=-2 .
\end{array}
$$

\section{REFERENCES}

[1] R. Verdone, D. Dardari, G. Mazzini, and A. Conti, Wireless Sensor and Actuator Networks: Technologies, Analysis and Design. Elsevier, 2008.

[2] I. F. Akyildiz, W. Su, Y. Sankarsubramanian, and E. Cayirci, "Wireless sensor networks: a survey," Comput. Netw., vol. 38, no. 4, pp. 393-422, Mar. 2002.

[3] J. Berger, V. D. Oliveira, and B. Sanso, "Objective Bayesian analysis of spatially correlated data," J. American Statistical Association, vol. 96, pp. 1361-1374, Dec. 2001

[4] M. Dong, L. Tong, and B. Sadler, "Impact of data retrieval pattern on homogeneous signal field reconstruction in dense sensor networks," IEEE Trans. Signal Process., vol. 54, no. 11, pp. 4352-4364, Nov. 2006.

[5] M. C. Vuran, O. B. Akan, and I. F. Akyildiz, "Spatio-temporal correlation: theory and applications for wireless sensor networks," Elsevier Comput. Netw., vol. 45, no. 3, pp. 245-259, 2004.

[6] D. Marco and D. Neuhoff, "Reliability vs. efficiency in distributed source coding for field-gathering sensor networks," in Proc. IEEE IPSN, Apr. 2004, pp. 161-168.

[7] J. Matamoros and C. Antón-Haro, "Bandwidth constraints in wireless sensor-based decentralized estimation schemes for Gaussian channels," in Proc. IEEE Global Conf. Commun., Dec. 2008, pp. 1-5. 
[8] J.-J. Xiao, S. Cui, Z.-Q. Luo, and A. J. Goldsmith, "Power scheduling of universal decentralized estimation in sensor networks," IEEE Trans. Signal Process., vol. 54, no. 2, pp. 431-422, Feb. 2006.

[9] A. Ribeiro and G. Giannakis, "Bandwidth-constrained distributed estimation for wireless sensor networks-part I: Gaussian case," IEEE Trans. Signal Process., vol. 54, no. 3, pp. 1131-1143, Mar. 2006.

[10] A. Ribeiro and G. B. Giannakis, "Bandwidth-constrained distributed estimation for wireless sensor networks-part II: unknown probability density function," IEEE Trans. Signal Process., vol. 54, no. 7, pp. 2784 2796, July 2006.

[11] P. Ishwar, A. Kumar, and K. Ramchandran, "Distributed sampling for dense sensor networks: a 'bit-conservation principle'," Lecture Notes Comput. Science, vol. 2634/2003, pp. 17-31, Jan. 2003.

[12] J. Matamoros and C. Antón-Haro, "Optimal network size and encoding rate for random field estimation with wireless sensor networks," in Proc. 3rd Int'l Workshop Computational Advances Multi-Sensor Adaptive Process., Dec. 2009.

[13] J. Fang and H. Li, "Distributed estimation of Gauss-Markov random fields with one-bit quantized data," IEEE Signal Process. Lett., vol. 17, no. 5, pp. 449-452, May 2010.

[14] D. Dardari, A. Conti, C. Buratti, and R. Verdone, "Mathematical evaluation of environmental monitoring estimation error through energyefficient wireless sensor networks," IEEE Trans. Mobile Comput., vol. 6, no. 7, pp. 790-802, July 2007.

[15] J. Matamoros and C. Antón-Haro, "Random field estimation with delayconstrained and delay-tolerant wireless sensor networks," EURASIP J. Wireless Commun. Netw., vol. 2010, pp. 1-13, May 2010.

[16] W. Gardner, Introduction to Random Processes: With Applications to Signals and Systems, 2nd edition. McGraw Hill, 1989.

[17] E. Masry, "Poisson sampling and spectral estimation of continuous-time processes," IEEE Trans. Inf. Theory, vol. 24, no. 2, pp. 173-183, Mar. 1978.

[18] F. Marvasti, "Signal recovery from nonuniform samples and spectral analysis on random nonuniform samples," in Proc. IEEE International Conf. Acoustics, Speech, Signal Process., vol. 11, Apr. 1986, pp. 16491652.

[19] N. S. Jayant and L. R. Rabiner, "The application of dither to the quantization of speech signals," Bell Syst. Tech. J., vol. 51, pp. 1293 1304, July/Aug. 1972.

[20] S. Cui, J. Xiao, A. Goldsmith, Z.-Q. Luo, and H. V. Poor, "Estimation diversity and energy efficiency in distributed sensing," IEEE Trans. Signal Process., vol. 55, no. 9, pp. 4683-4695, Sep. 2007.

[21] J. Li and G. AlRegib, "Rate-constrained distributed estimation in wireless sensor networks," IEEE Trans. Signal Process., vol. 55, no. 5, pp. 1634-1643, May 2007

[22] R. Corless, G. Gonnet, D. Hare, and D. Jeffrey, "On the Lambert $W$ function," Advances Computational Mathematics, vol. 5, pp. 329-359, 1996.

[23] S. Boyd and L. Vandenberghe, Convex Optimization. Cambridge University Press, 1993.

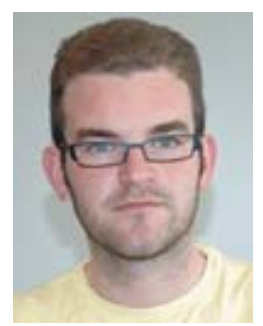

Javier Matamoros (M'10) received the M.Sc. and the Ph.D. degrees in Telecommunications Engineering from the Polytechnic University of Catalonia in 2005 and 2010 respectively. From February 2005 until August 2005 he was with the WMC (Wireless Mobile and Communication) group of TUDelft (Delft University of Technology). In January 2006, he was granted by the CTTC and, in January 2008, by the Spanish Ministry of Science and Innovation to pursuit his Ph.D degree. From August 2010, he holds a research associate position at the CTTC in the Intelligent Energy (IQe) area. His primary research interests include Wireless Sensor Networks, Information Theory, Distributed Signal Processing and Smart Grids.

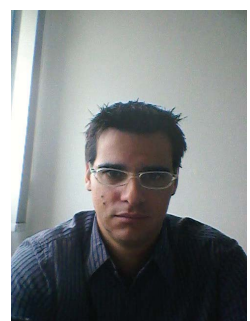

Dr. Flavio Fabbri received the M.Sc. and $\mathrm{PhD}$ degrees in Telecommunication Engineering from the University of Bologna, Italy, in 2007 and 2011, respectively. He has been involved in research activities in wireless communication networks, specifically wireless ad hoc and sensor networks, since 2007. He is currently a PostDoc researcher with the Networked Embedded Systems group at the University of Duisburg-Essen, Germany. He is actively involved in the European Network of Excellence on Cooperating Objects, CONET, and has been a visitor of iNETS group at RWTH Aachen University, Germany, and WNCG group at University of Austin, TX, USA.

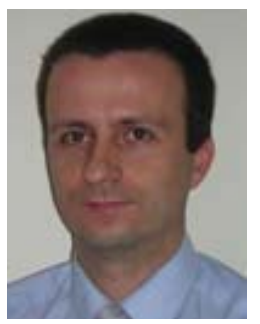

Carles Antón-Haro (M'99-SM'03) received his $\mathrm{Ph} . \mathrm{D}$. degree in Telecommunications engineering from the Technical University of Catalonia (UPC) in 1998 (cum-laude). In 1999, he joined Ericsson Spain, where he participated in rollout projects of $2 \mathrm{G}$ and $3 \mathrm{G}$ mobile networks. Currently, he is with the Centre Tecnologic de Telecomunicacions de Catalunya (CTTC) as a Director of International Relations and Senior Researcher. His research interests are in the area of signal processing for communications, this including MIMO, opportunistic communications, array signal processing, Smart Grids, M2M communications, and wireless sensor networks. He has published +20 technical papers in IEEE journals as well as over 65 papers in international and national conferences. Dr. Anton-Haro is a Senior Member of the IEEE.

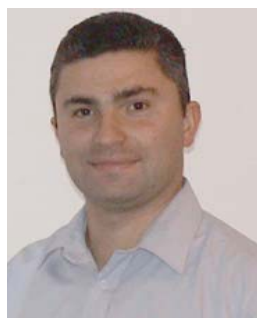

Davide Dardari (Senior Member, IEEE) received the Laurea degree in electronic engineering (summa cum laude) and the $\mathrm{PhD}$ degree in electronic engineering and computer science from the University of Bologna, Italy, in 1993 and 1998, respectively. Currently, he is Associate Professor at the University of Bologna at Cesena, Cesena (FC), Italy. He is Research Affiliate with WiLAB and Wireless Communications Group at LIDS, Massachusetts Institute of Technology (MIT). His actual research interests are in ultrawide bandwidth communication and localization, wireless sensor networks, OFDM systems. He published more than 100 technical papers and played several important roles in various National and European Projects. He is co-author of the book Wireless Sensor and Actuator Networks: Enabling Technologies, Information Processing and Protocol Design (NewYork: Elsevier, 2008). Davide Dardari is the current Chair of the Radio Communications Committee of the IEEE Communication Society. He is co-general chair of the IEEE International Conference on UWB (ICUWB) 2011. He was co-chair of the Wireless Communications Symposium of the 2007 IEEE International Conference on Communications (ICC) and of the IEEE ICUWB 2006. He was Lead Editor for the EURASIP Journal on Advances in Signal Processing (Special Issue on Cooperative Localization in Wireless Ad Hoc and Sensor Networks), Guest Editor for the Proceedings of the IEEE (Special Issue on UWB Technology \& Emerging Applications), Guest Editor for Physical Communication Journal (ELSEVIER) (Special Issue on Advances in Ultra-Wideband Wireless Communications), and Guest Editor for EURASIP (Hindawi) Journal on Wireless Communications and Networking (Special Issue on Signal Processing-assisted Protocols and Algorithms for Cooperating Objects and Wireless Sensor Networks). Currently, he is an Associate Editor for IEEE TRANS ACTIONS ON WIRELESS COMMUNICATIONS. 